\title{
Support Properties of the Free Measure for Boson Fields
}

\author{
M. Reed and L. Rosen \\ Mathematics Department, Princeton University, Princeton, N.J., \\ and Mathematics Department, University of Toronto, Toronto, Canada
}

Received November 6, 1973

\begin{abstract}
Let $\mu$ be the measure on $\mathscr{S}^{\prime}\left(\mathbb{R}^{d}\right)$ corresponding to the Gaussian process with mean zero and covariance $\left(f,(-\Delta+1)^{-1} g\right)$ on $\mathscr{S}\left(\mathbb{R}^{d}\right)$. It is proven that the set

$$
\left(-\Delta_{d-1}+1\right)^{d / 4-\frac{1}{2}+\alpha}\left(1+x^{2}\right)^{d / 4}\left[\log \left(2+x^{2}\right)\right]^{\beta} L^{2}\left(\mathbb{R}^{d}\right)
$$

has $\mu$ measure one if $\alpha>0$ and $\beta>\frac{1}{2}$ and $\mu$ measure zero if $\alpha>0$ and $\beta<\frac{1}{2}$; here $\Delta_{d-1}$ is the Laplacian in any $d-1$ dimensions when $d>1$ and $\Delta_{0}=\Delta$.
\end{abstract}

\section{§ 1. Introduction}

Nelson [11] has shown that to every generalized stochastic process $\phi$ over $\mathscr{S}\left(\mathbb{R}^{d}\right)$ satisfying the axioms of a Euclidean Markov field theory there corresponds in a natural way a relativistic Boson field theory satisfying the Wightman axioms. In particular, the Gaussian process of mean zero with covariance $\langle\phi(f) \phi(g)\rangle=\left(f,(-\Delta+1)^{-1} g\right)$ corresponds to the free Boson field of mass 1 in $d$-dimensional space-time [12]. By Minlos' theorem $[6,8]$ this "free process" can be realized on $\mathscr{S}^{\prime}\left(\mathbb{R}^{d}\right)$, the topological dual of the nuclear space $\mathscr{S}\left(\mathbb{R}^{d}\right)$. That is, there is a Borel measure $\mu$ on $\mathscr{S}^{\prime}\left(\mathbb{R}^{d}\right)$ so that

$$
e^{-\frac{1}{2}\left(f,(-\Delta+1)^{-1} f\right)}=\int_{\mathscr{S}^{\prime}\left(\mathbb{R}^{d}\right)} e^{i\langle q, f\rangle} d \mu(q)
$$

for all $f \in \mathscr{S}\left(\mathbb{R}^{d}\right)$. In this realization, the free process itself is given by the function $\phi(f): q \rightarrow\langle q, f\rangle$ for $q \in \mathscr{S}^{\prime}$.

The processes corresponding to interacting Boson theories are usually constructed by taking limits of non-Gaussian perturbations of the "free measure" $\mu$ (see, for example, [10]). The properties of the free measure play an important role in this procedure $[4,7]$. Thus it seems useful to study the free process in order to gain insight into and develop analytic tools for the more complicated interacting processes.

In this paper we investigate the support properties of $\mu$ by elementary methods. The point is that $\mathscr{S}^{\prime}$ is an unnecessarily large space in which to 
realize the free process. A space not much larger than the dual $\mathscr{H}_{+1}$ of the Hilbert space $\mathscr{H}_{-1}$ should suffice. Here $\mathscr{H}_{\alpha}$ is the Hilbert space completion of $\mathscr{S}\left(\mathbb{R}^{d}\right)$ in the norm $\|f\|_{\mathscr{H}_{\alpha}}=\left\|P^{\alpha} f\right\|$ where $P=(-\Delta+1)^{\frac{1}{2}}$. Let $P_{1}=\left(-\Delta_{d-1}+1\right)^{\frac{1}{2}}$ and $Q=\left(2+x^{2}\right)^{\frac{1}{2}}$ where $\Delta_{d-1}$ is the Laplacian in any $d-1$ variables. The result of this paper says that the set

$$
S=P_{1}^{d / 2-1+\alpha} Q^{d / 2}(\log Q)^{\beta} L^{2}\left(\mathbb{R}^{d}\right)
$$

has $\mu$ measure 1 for any $\alpha>0$ and $\beta>\frac{1}{2}$ and that this result is globally sharp in the sense that $S$ has $\mu$ measure 0 for any $\alpha>0$ and $\beta<\frac{1}{2}$. In the case $d=1$, all the statements in this paper involving $P_{1}$ should be interpreted with $P_{1}$ replaced by $P$. By "measurable" we always mean measurable with respect to the smallest $\sigma$-algebra containing the cylinder sets. One can see that $S$ is measurable as follows: Let $T$ be a bijective continuous map of $\mathscr{S}\left(\mathbb{R}^{d}\right)$ onto itself and let $\left\{h_{n}\right\}$ be an orthonormal basis for $L^{2}\left(\mathbb{R}^{d}\right)$ with $h_{n} \in \mathscr{S}\left(\mathbb{R}^{d}\right)$. Then $q=T^{*} f, f \in L^{2}\left(\mathbb{R}^{d}\right)$, if and only if $q\left(T^{-1} h_{n}\right)=\left(f, h_{n}\right)$ satisfies:

$$
G(q) \equiv \sum^{\infty}\left|q\left(T^{-1} h_{n}\right)\right|^{2}<\infty .
$$

Now if $G_{N}(q)=\sum^{N}\left|q\left(T^{-1} h_{n}\right)\right|^{2}$, then $G_{N}$ is measurable for each $N$ so $G=\lim \sup G_{N}$ is measurable. Thus $T^{*} L^{2}\left(\mathbb{R}^{d}\right)$ is a measurable subset of $\mathscr{P}^{\prime}\left(\mathbb{R}^{d}\right)$.

The local measure one result, i.e. the fact that locally the "paths" are almost surely in $P_{1}^{d / 2-1+\alpha} L^{2}$, is a special case of recent work of Cannon [2]. Cannon also remarks that the local result is sharp in the sense that $P_{1}^{d / 2-1} L^{2}$ is a set of measure zero. Global and local results for the free process, expressed in terms of lim sup properties (rather than $L^{2}$ properties), have been obtained by Colella and Lanford [3].

Acknowledgements. Conversations and correspondence with John Cannon and Oscar Lanford originally stimulated our interest in the subject. We also wish to thank Bill Faris and Barry Simon for useful conversations.

Note added in Proof. After submission of the manuscript, the authors learned from O. E. Lanford, III, that he has an easier generalization of the same result which relies only on the Gaussian nature of the process and does not use the Kolmogorov three series theorem or directly the asymptotic independence.

\section{$\S$ 2. Sets of Measure One}

We begin by stating Minlos' Theorem [6,8] for the case of Gaussian processes. The topological dual of a nuclear space $E$ will be denoted by $E^{*}$ and the natural pairing between $E$ and $E^{*}$ by $\langle\cdot, \cdot\rangle$. The superscript * always indicates the dual space with respect to this pairing. 
Theorem (Minlos). Let $(\cdot, \cdot)_{0}$ and $(\cdot, \cdot)_{1}$ be continuous scalar products on a nuclear space $E$ such that $\|f\|_{0} \leqq c\|f\|_{1}$ for some constant $c$ and all $f \in E$. Let $H_{0}$ and $H_{1}$ denote the Hilbert space completions of $E$ with respect to $\|\cdot\|_{0}$ and $\|\cdot\|_{1}$. Suppose that the natural injection $H_{1} \rightarrow H_{0}$ is a Hilbert-Schmidt operator. Then $H_{1}^{*}$ is a set of measure one for the Gaussian process $\phi(f)(\cdot)=\langle\cdot, f\rangle$ over $E$ with covariance $\langle\phi(f) \phi(g)\rangle$ $=(f, g)_{0}$.

To say that the natural injection $H_{1} \rightarrow H_{0}$ is Hilbert-Schmidt is equivalent to saying that there is a Hilbert-Schmidt operator $T$ on $H_{0}$ such that $H_{1}$ is the set $T H_{0}$ with norm $\|f\|_{1}=\left\|T^{-1} f\right\|_{0}$. It is convenient to consider the following Hilbert-Schmidt operator $\left(P, P_{1}, Q\right.$ are defined in $\S 1)$ :

Lemma 1. Define $\tilde{T}=P^{-1} P_{1}^{-\alpha} Q_{\beta}^{-1}$ where $Q_{\beta}=Q^{d / 2} \log ^{\beta} Q$. Then $\tilde{T}$ is Hilbert-Schmidt on $L^{2}\left(\mathbb{R}^{d}\right)$ if and only if $\alpha>d / 2-1$ and $\beta>\frac{1}{2}$.

We defer the proof until the end of this section. The lemma is a special case of the general principle that an operator on $L^{2}\left(\mathbb{R}^{d}\right)$ is HilbertSchmidt if it consists of slightly more than $d / 2$ powers of each of $P^{-1}$ and $Q^{-1}$. Applying Minlos' Theorem we thus obtain:

Theorem 1. For any $\alpha>d / 2-1$ and $\beta>\frac{1}{2}, S=P_{1}^{\alpha} Q_{\beta} L^{2}\left(\mathbb{R}^{d}\right)$ is a set of measure one for the Gaussian process over $\mathscr{S}\left(\mathbb{R}^{d}\right)$ with covariance $\left(f,(-\Delta+1)^{-1} g\right)$.

Remark. When $d=1$, we take $S=P^{\alpha} Q_{\beta} L^{2}$ (see the discussion following the proof).

Proof. Since $P$ is a unitary map of $L^{2}\left(\mathbb{R}^{d}\right)$ into $\mathscr{H}_{-1}$ an operator $T$ on $\mathscr{H}_{-1}$ will be Hilbert-Schmidt if and only if $\tilde{T}=P^{-1} T P$ is HilbertSchmidt on $L^{2}$. Thus by Lemma $1, T=P_{1}^{-\alpha} Q_{\beta}^{-1} P^{-1}$ is Hilbert-Schmidt on $\mathscr{H}_{-1}$. According to Minlos' Theorem, $H_{1}^{*}$ is a set of $\mu$ measure one, where $H_{1}=T \mathscr{H}_{-1}$ with norm $\|f\|_{1}=\left\|T^{-1} f\right\|_{\mathscr{H}_{-1}}=\left\|Q_{\beta} P_{1}^{\alpha} f\right\|$. That is, $H_{1}^{*}$ is the completion of $\mathscr{S}$ in the norm $\left\|Q_{\beta}^{-1} P_{1}^{-\alpha} f\right\|$, whose elements we denote by $S=P_{1}^{\alpha} Q_{\beta} L^{2}$.

An examination of the proofs of Lemma 1 and Theorem 1 reveals that there is considerable flexibility in the choice of $H_{1}$. For instance the conclusion of Theorem 1 holds equally as well for the sets

$$
P^{\alpha} Q_{\beta} L^{2}, \quad P_{1}^{d / 2-1} \log ^{\gamma} P_{1} Q_{\beta} L^{2} \ldots
$$

where $\alpha>d / 2-1, \beta>\frac{1}{2}, \gamma>\frac{1}{2}$. The stronger statements (involving $P_{1}$ rather than $P$ ) show that it is sufficient to smooth in only $d-1$ variables in order that the paths be locally in $L^{2}$ almost surely.

Proof of Lemma 1. The kernel of the integral operator $\tilde{T}$ is $\tilde{T}(x, y)$ $=\hat{h}(x-y) g(y)$ where

$$
g(y)=\left(2+y^{2}\right)^{-d^{\prime} 4}\left(\log \left(2+y^{2}\right)^{\frac{1}{2}}\right)^{-\beta}
$$


and $h$ is the Fourier transform of

$$
h(p)=\left(1+p^{2}\right)^{-\frac{1}{2}}\left(1+\sum_{k=1}^{d-1} p_{k}^{2}\right)^{-\alpha / 2}
$$

where without loss of generality we have chosen $\Delta_{d-1}$ to be the Laplacian in the first $d-1$ variables. Thus by the Plancherel Theorem the HilbertSchmidt norm of $\tilde{T}(x, y)$ is equal to $\|h\| \cdot\|g\|$.

It remains to verify that $h \in L^{2}$ and $g \in L^{2}$ if and only if $\alpha>d / 2-1$ and $\beta>\frac{1}{2}$ respectively. Changing to spherical coordinates in $\mathbb{R}^{d-1}$ we have

$$
\begin{aligned}
\|h\|^{2} & =\iiint \frac{d p_{n} r^{d-2} d r d \Omega}{\left(1+r^{2}+p_{n}^{2}\right)\left(1+r^{2}\right)^{\alpha}} \\
& =\operatorname{const} \int_{0}^{\infty} \frac{r^{d-2} d r}{\left(1+r^{2}\right)^{\alpha+\frac{1}{2}}}
\end{aligned}
$$

which is finite if and only if $\alpha>d / 2-1$.

Similarly, by changing to polar coordinates in $\mathbb{R}^{d}$,

$$
\|g\|^{2}=\text { const } \int_{0}^{\infty} \frac{r^{d-1} d r}{\left(2+r^{2}\right)^{d / 2}\left(\log \left(2+r^{2}\right)^{\frac{1}{2}}\right)^{2 \beta}}
$$

which is finite if and only if $\beta>\frac{1}{2}$ as can be seen by the change of variable $r=e^{s}$.

\section{§ 3. Sets of Measure Zero}

In the last section we proved that if $\alpha>d / 2-1$ then $S=P_{1}^{\alpha} Q_{\beta} L^{2}\left(\mathbb{R}^{d}\right)$ is a set of measure one for the Gaussian process over $\mathscr{S}\left(\mathbb{R}^{d}\right)$ with covariance $\left(f,(-\Delta+1)^{-1} g\right)$ when $\beta>\frac{1}{2}$. In this section we prove that $S$ is contained in a set of measure zero when $\beta<\frac{1}{2}$. This amounts to the same thing as showing that for $\beta<\frac{1}{2}, Q_{\beta} L^{2}\left(\mathbb{R}^{d}\right)$ is contained in a set of measure zero for the Gaussian process $\phi_{1}$ over $\mathscr{S}\left(\mathbb{R}^{d}\right)$ with covariance

$$
\left\langle\phi_{1}(f) \phi_{1}(g)\right\rangle=(f, g)_{1} \equiv\left(f, P_{1}^{-2 \alpha} P^{-2} g\right) .
$$

The idea of the proof is simple: namely, we shall find a sequence of functions $g_{n} \in \mathscr{S}\left(\mathbb{R}^{d}\right)$ which are orthogonal in the inner product $(\cdot, \cdot)_{1}$ so that the random variables $\psi_{n} \equiv\left|\phi_{1}\left(g_{n}\right)\right|$ are independent and which are chosen so that

$$
\begin{aligned}
& \Sigma \psi_{n}(q)<\infty \text { for all } q \in Q_{\beta} L^{2} \\
& \Sigma E \psi_{n}^{c}=\infty
\end{aligned}
$$

where $E$ denotes expectation with respect to the underlying measure $\mu_{1}$ for the process $\phi_{1}$, and $\psi^{c}$ is the truncation $\psi^{c}=\psi$ or 0 according as $|\psi|<c$ or $|\psi| \geqq c$. We can then conclude by the Kolmogorov Three Series Theorem [9] that the set $Q_{\beta} L^{2}$ is a set of $\mu_{1}$ measure zero. The 
difficulty which arises in this argument is that the natural choices for the $g_{n}$, namely functions whose supports move out to infinity, are not orthogonal since $\left(g_{n}, g_{m}\right)_{1} \neq 0$ even though $g_{n}$ and $g_{m}$ have disjoint supports. However $\left(g_{n}, g_{m}\right)_{1}$ goes exponentially to zero as the supports of $g_{n}$ and $g_{m}$ separate, and we use this familiar exponential decay [7] to modify the "natural" $g_{n}$ by Gram-Schmidt orthogonalization.

We begin by introducing some terminology. $|\cdot|$ will denote the usual norm on $\mathbb{R}^{d}$ while $|\cdot|_{1}$ will denote the norm $|x|_{1}=\left|x_{1}\right|+\cdots+\left|x_{d}\right|$. The symbol $n=\left(n_{1}, \ldots, n_{d}\right)$ will always denote a $d$-tuple of integers, i.e. $n \in \mathbb{Z}^{d}$. We order such $d$-tuples by starting with $0=(0,0, \ldots, 0)$, then ordering the $n$ so that $|n|_{1}=1$, then the $n$ so that $|n|_{1}=2$ and so forth; $n^{-}$denotes the predecessor of $n$. Thus the summation symbol $\sum_{n=0}^{K^{-}}$is unambiguous. Throughout this section $\chi$ denotes a fixed $C_{0}^{\infty}$ function with support in the $d$-cube of side length one with centre at the origin normalized so that $\|\chi\|_{1}=1$. Letting $K$ be a large positive integer (to be chosen later) we set

$$
\chi_{n}(x)=\chi(x-K n) .
$$

Notice that for all $n,\left\|\chi_{n}\right\|_{1}$ and $\left\|\chi_{n}\right\|$ (the $L^{2}$-norm) are constant. Finally, we define $\tilde{\chi}_{n}$ to be the unnormalized Gram-Schmidt orthogonalization of $\chi_{n}$, where $\chi_{0}=\chi$ :

$$
\tilde{\chi}_{n}=\chi_{n}-\sum_{j=0}^{n^{-}} c_{j}^{n} \chi_{j}
$$

The following two lemmas (proven at the end of the section) tell us how to choose $K$.

Lemma 2. Given any $a>0$, we can choose $K$ so large that

$$
\left|\left(\chi_{n}, \chi_{m}\right)_{1}\right| \leqq a^{|n-m|_{1}}
$$

for all $m$ and $n$.

Lemma 3. For a small enough, there are constants $e>0$ and $D$ (independent of $n$ and $j$ ) so that

(a) $\left\|\tilde{\chi}_{n}\right\|^{2} \geqq e^{2}$.

(b) $\sum_{n>j}\left|c_{j}^{n}\right| \leqq D$.

Theorem 2. If $\alpha>d / 2-1$ and $\beta<\frac{1}{2}$ then $S=P_{1}^{\alpha} Q_{\beta} L^{2}\left(\mathbb{R}^{d}\right)$ is contained in a set of measure zero for the Gaussian process over $\mathscr{S}\left(\mathbb{R}^{d}\right)$ with covariance $\left(f,(-\Delta+1)^{-1} g\right)$.

Remark. Again, when $d=1$, we replace $P_{1}$ by $P$ in the theorem.

Proof. As mentioned above it is sufficient to show that $Q_{\beta} L^{2}\left(\mathbb{R}^{d}\right)$ is a set of measure zero for the Gaussian process with covariance (3.1). We 
define

$$
\begin{aligned}
& \gamma_{n}=\left(2+n^{2}\right)^{d / 4}\left[\log \left(2+n^{2}\right)\right]^{\beta} \\
& \tau_{n}=\left(2+n^{2}\right)^{d / 4}\left[\log \left(2+n^{2}\right)\right]^{3 / 4-\beta / 2} .
\end{aligned}
$$

Now we choose $K$ large enough so that the conclusion of Lemma 3 holds and define

$$
g_{n}=\frac{\tilde{\chi}_{n}}{\gamma_{n} \tau_{n}} \quad \text { and } \quad \psi_{n}=\left|\phi_{1}\left(g_{n}\right)\right|
$$

Let $q \in Q_{\beta} L^{2}\left(\mathbb{R}^{d}\right)$, i.e. $q=\left(2+x^{2}\right)^{d / 4}\left[\log \left(2+x^{2}\right)\right]^{\beta} f(x)$ where $f$ is in $L^{2}\left(\mathbb{R}^{d}\right)$. In the following calculation $c$ denotes various universal constants (independent of $n$ ) and $\square_{n}$ denotes the $d$-cube of side length one with centre at $n$. Now

$$
\begin{aligned}
\psi_{n}(q) & \leqq \int \frac{\left|Q_{\beta}(y)\right||f(y)|}{\gamma_{n} \tau_{n}}\left|\chi_{n}-\sum_{j=0}^{n^{-}} c_{j}^{n} \chi_{j}\right| d y \\
& \leqq \frac{c}{\tau_{n}}\left\{\int_{\square_{n}}\left|f(y) \chi_{n}(y)\right| d y+\sum_{j=0}^{n^{-}}\left|c_{j}^{n}\right| \int_{\square_{j}}\left|f(y) \chi_{j}(y)\right| d y\right\} \\
& \leqq \frac{c}{\tau_{n}}\left\{\left(\int_{\square_{n}}|f|^{2} d y\right)^{\frac{1}{2}}+\sum_{j=0}^{n^{-}}\left|c_{j}^{n}\right|\left(\int_{\square_{j}}|f|^{2} d y\right)^{\frac{1}{2}}\right\} .
\end{aligned}
$$

Thus,

$$
\sum_{n} \psi_{n}(q) \leqq c \sum_{n} \frac{1}{\tau_{n}}\left(\int_{\square_{n}}|f|^{2} d y\right)^{\frac{1}{2}}+c \sum_{n} \sum_{j=0}^{n^{-}} \frac{\left|c_{j}^{n}\right|}{\tau_{n}}\left(\int_{\square_{j}}|f|^{2} d y\right)^{\frac{1}{2}} .
$$

The first term on the right is less than infinity since $\left\{\frac{1}{\tau_{n}}\right\} \in \ell^{2}$ by the proof of Lemma 1 and $\left\{\left(\int_{\square_{n}}|f|^{2} d y\right)^{\frac{1}{2}}\right\} \in \ell^{2}$ since the $\square_{n}$ are disjoint. To handle the second term we rewrite it:

$$
\begin{aligned}
& c \sum_{j=0}^{\infty} \sum_{n>j} \frac{\left|c_{j}^{n}\right|}{\tau_{n}}\left(\int_{\square_{j}}|f|^{2} d y\right)^{\frac{1}{2}} \\
& \leqq c D \sum_{j=0}^{\infty} \frac{1}{\tau_{j}}\left(\int_{\square_{j}}|f|^{2} d y\right)^{\frac{1}{2}} \quad \text { (by Lemma 3) } \\
& <\infty
\end{aligned}
$$

by the same reasoning as above. Thus,

$$
\sum_{n} \psi_{n}(q)<\infty \text { for all } q \in Q_{\beta} L^{2}\left(\mathbb{R}^{d}\right) .
$$

On the other hand, for each $n, \phi_{1}\left(g_{n}\right)$ is a Gaussian random variable of mean zero and covariance

$$
\sigma_{n}=\frac{\left\|\tilde{\chi}_{n}\right\|_{1}^{2}}{\left(\gamma_{n} \tau_{n}\right)^{2}} \geqq\left(\frac{e}{\gamma_{n} \tau_{n}}\right)^{2}
$$


by Lemma 3 [Part (a)]. Since the $\phi_{1}\left(g_{n}\right)$ are Gaussian and orthogonal they are independent and thus so are the $\psi_{n}$. Now

$$
\begin{aligned}
E \psi_{n}^{c} & \equiv \frac{1}{\sqrt{2 \pi \sigma_{n}}} \int_{|\lambda| \leqq c}|\lambda| e^{-\lambda^{2} / 2 \sigma_{n}^{2}} d \lambda \\
& =\frac{\sqrt{\sigma_{n}}}{\sqrt{2 \pi}} \int_{|s| \leqq \frac{c}{\sqrt{\sigma_{n}}}}|s| e^{-s^{2} / 2} d s \\
& \geqq \frac{\sqrt{\sigma_{n}}}{\sqrt{2 \pi}},
\end{aligned}
$$

for $n$ large enough since $\sigma_{n} \rightarrow 0$.

By the proof of Lemma $1, \sum_{n} \frac{1}{\cdot \gamma_{n} \tau_{n}}=\infty$ so we conclude that

$$
\sum_{n} E \psi_{n}^{c}=\infty \text {. }
$$

Thus, by the Kolmogorov three series theorem [9], the set of $q \in \mathscr{S}\left(\mathbb{R}^{d}\right)$ so that $\sum_{n} \psi_{n}(q)<\infty$ has measure zero. Since we have already shown this inequality for $q \in Q_{\beta} L^{2}\left(\mathbb{R}^{d}\right)$ the proof is finished.

It remains to prove Lemmas 2 and 3.

Proof of Lemma 2. If $\gamma>0$, the operator $P^{-\gamma}$ acts by convolution with a distribution $k_{\gamma, d}$ which is the Fourier transform of $\left(1+p^{2}\right)^{-\gamma / 2}$. By [5],

$$
k_{\gamma, d}(x)=(2 \pi)^{-d / 2} 2^{1-\gamma / 2} \Gamma(\gamma / 2)^{-1}|x|^{\frac{\gamma-d}{2}} K_{\frac{d-\gamma}{2}}(|x|)
$$

where $K_{v}$ is the modified Bessel function. Thus $k_{\gamma, d}$ is $C^{\infty}$ except possibly at $x=0$ and its behaviour at zero and infinity is given by (see, for instance, [1]):

$$
\begin{aligned}
& \text { as }|x| \rightarrow 0, \quad k_{\gamma, d}(x)=\left\{\begin{array}{lll}
O\left(|x|^{\gamma-d}\right) & \text { if } \gamma<d \\
O(\log |x|) & \text { if } & \gamma=d \\
O(1) & \text { if } & \gamma>d
\end{array}\right. \\
& \text { as } \quad|x| \rightarrow \infty, \quad k_{\gamma, d}(x)=O\left(|x|^{\frac{\gamma-d-1}{2}} e^{-|x|}\right) .
\end{aligned}
$$

Therefore $k_{2, d}$ is integrable near zero and

$$
\begin{aligned}
\left|\left(P^{-2} \chi_{n}\right)(x)\right| & =\left|\int k_{2, d}(x-y) \chi(y-K n) d y\right| \\
& \leqq c e^{-|x-K n|_{1} / V d}
\end{aligned}
$$

where $c$ will denote various constants independent of $x, m$, and $n$ and where we have used the relation $|x|_{1} \leqq \sqrt{d}|x|$. 
Similarly we have

$$
\begin{aligned}
\left|\left(P_{1}^{-2 \alpha} \chi_{m}\right)(x)\right| & =\left|\int_{\mathbb{R}^{d-1}} k_{2 \alpha, d-1}(\hat{x}-\hat{y}) \chi\left(\hat{y}-K \hat{m}, x_{d}-K m_{d}\right) d \hat{y}\right| \\
& \leqq c e^{-|\hat{x}-K \hat{m}|_{1} / \sqrt{d}} \bar{\chi}\left(x_{d}-K m_{d}\right)
\end{aligned}
$$

where $\hat{x}=\left(x_{1}, \ldots, x_{d-1}\right)$ and $\bar{\chi}$ is the characteristic function of $\left[-\frac{1}{2}, \frac{1}{2}\right]$. By the triangle inequality

$$
\begin{aligned}
|x-K n|_{1}+|\hat{x}-K \hat{m}|_{1} & \geqq \frac{1}{2}\left(|K \hat{n}-K \hat{m}|_{1}+|\hat{x}-K \hat{n}|_{1}+|\hat{x}-K \hat{m}|_{1}\right) \\
& +\left|x_{d}-K n_{d}\right|
\end{aligned}
$$

so that by (3.2) and (3.3)

$$
\begin{aligned}
\left|\left(\chi_{m}, P_{1}^{-2 \alpha} P^{-2} \chi_{n}\right)\right| \leqq & c e^{-K|\hat{n}-\hat{m}|_{1} / 2 V \bar{d}} \int_{\mathbb{R}^{d-1}} e^{-\left(|\hat{x}-K \hat{n}|_{1}+|\hat{x}-K \hat{m}|_{1}\right) / 2 \sqrt{d}} d \hat{x} \\
& \cdot \int \bar{\chi}\left(x_{d}-K m_{d}\right) e^{-\left|x_{d}-K n_{d}\right| / 2 V d} d x_{d} \\
\leqq & c e^{-K|n-m|_{1} / 2 V d} .
\end{aligned}
$$

This proves Lemma 2.

To prove Lemma 3 we first establish a preliminary lemma which expresses the fact that if the components of a matrix $A$ decay exponentially away from the diagonal then the same is true of $A^{-1}$, independently of the size of $A$ :

Lemma 4. Let $S$ be a finite subset of $\mathbb{Z}^{d}$ and let $A$ be a matrix indexed by $S$ which satisfies $A_{i i}=1$ and

$$
\left|A_{i j}\right| \leqq a^{|i-j|_{1}}
$$

where $a>0$. Then there are constants $a_{1}$ and $b$ (independent of the number of points $N$ in $S$ ) such that for $a \leqq a_{1}$,

$$
\left|A_{i j}^{-1}\right| \leqq b(4 a d)^{|i-j|_{1}}
$$

Proof. It is sufficient to prove the lemma if $S$ is a rectangular array of adjacent points in $Z^{d}$. For if not, simply embed $S$ in such a rectangle $\tilde{S}$ and extend $A$ to a matrix $\tilde{A}$ indexed by $\tilde{S}$ by $\tilde{A}=A \oplus I$, i.e. $\tilde{A}_{i j}=A_{i j}$ if $i, j \in S$ and $\tilde{A}_{i j}=\delta_{i j}$ otherwise. Then $\tilde{A}$ satisfies (3.4) and so $\tilde{A}^{-1}$ satisfies (3.5). But $\tilde{A}^{-1}=A^{-1} \oplus I$ so that $A^{-1}$ also satisfies (3.5).

To prove the lemma in this case, we introduce another matrix $R$ also indexed by $S$ defined by $R_{i j}=1$ if $|i-j|_{1}=1$ and $R_{i j}=0$ otherwise. $R^{k}$ has the following properties:

$$
\begin{array}{ll}
\left(R^{k}\right)_{i j}=0 \quad \text { if } & k<|i-j|_{1}, \\
\left(R^{k}\right)_{i j} \geqq 1 \quad \text { if } & k=|i-j|_{1}, \\
\left(R^{k}\right)_{i j} \leqq(2 d)^{k} & \text { for all } i, j, k .
\end{array}
$$

These properties follow immediately from the interpretation of $\left(R^{k}\right)_{i j}$ as the number of ways of joining $i$ and $j$ by lines of length $k$ that are formed 
by segments of length 1 connecting points of $S$. Let $\|A\|$ denote the norm of the matrix $A$ corresponding to the supremum norm for vectors, i.e., $\|A\|=\max _{i} \sum_{j}\left|A_{i j}\right|$. Then also $\left\|R^{k}\right\| \leqq(2 d)^{k}$ since each row of $R$ contains at most $2 d$ ones.

Now consider the matrix $B=I-a R$. For $a<(2 d)^{-1}, B^{-1}=\sum_{0}^{\infty}(a R)^{k}$ converges in norm and therefore componentwise. Since $R$ has nonnegative elements, it follows from (3.7) that

$$
\left(B^{-1}\right)_{i j} \geqq a^{|i-j|_{1}} .
$$

We now estimate $A^{-1}$ by means of the Neumann series $A^{-1}$ $=\sum_{k=0}^{\infty}(I-A)^{k}$. From (3.4) it follows that

$$
\|I-A\| \leqq a \sum_{n=0}^{\infty} a^{|n|_{1}} .
$$

Thus for $a$ sufficiently small (independently of $N$ ) the Neumann series converges so

$$
\begin{aligned}
\left|\left(A^{-1}\right)_{i j}\right| & \leqq \sum_{k=0}^{\infty}\left|\left((I-A)^{k}\right)_{i j}\right| \\
& \leqq \sum_{k=0}^{\infty}\left(\left(B^{-1}-I\right)^{k}\right)_{i j}
\end{aligned}
$$

by (3.4) and (3.9). The series $\sum_{k=0}^{\infty}\left(B^{-1}-I\right)^{k}$ converges for $a$ sufficiently small (independently of $N$ ). In fact,

$$
\begin{aligned}
\sum_{k=0}^{\infty}\left(B^{-1}-I\right)^{k} & =\left(2 I-B^{-1}\right)^{-1} \\
& =I+a R(I-2 a R)^{-1} \\
& =I+\frac{1}{2} \sum_{k=1}^{\infty}(2 a R)^{k}
\end{aligned}
$$

which converges for $4 a d<1$. Thus if $i \neq j$ we conclude from (3.6), (3.8), (3.10) and (3.11) that

$$
\begin{aligned}
\left|\left(A^{-1}\right)_{i j}\right| & \leqq \frac{1}{2} \sum_{k=\sum_{i-j}}^{\infty}\left|\left((2 a R)^{k}\right)_{i j}\right| \\
& \leqq \frac{1}{2} \sum_{k=|i-j|_{1}}^{\infty}(4 a d)^{k} \\
& \leqq b(4 a d)^{|i-j|_{1}}
\end{aligned}
$$

where $b$ is independent of $N$.

Proof of Lemma 3. Define $A_{i j}=\left(\chi_{i}, \chi_{j}\right)_{1}$ and choose $K$ large enough by Lemma 2 so that $\left|A_{i j}\right| \leqq a^{|i-j|_{1}}$ where $a \leqq a_{1}$ with $a_{1}$ given by Lemma 4 . The coefficients $c_{j}^{n}$ are determined by the equations

$$
\sum_{j=0}^{n-} A_{i j} c_{j}^{n}=A_{i n}, \quad i=0, \ldots, n^{-} .
$$


That is, $c^{\vec{n}}=\left(A_{n}\right)^{-1} \vec{a}^{(n)}$ where $\vec{c}^{n}=\left(c_{0}^{n}, \ldots, c_{n^{-}}^{n}\right), \vec{a}^{(n)}=\left(A_{0 n}, \ldots, A_{n^{-} n}\right)$ and $A_{n}$ is the matrix $\left\{A_{i j}\right\}$ where $i, j=0, \ldots, n^{-}$. By Lemma 4

Thus

$$
\left|c_{j}^{n}\right| \leqq \sum_{i=0}^{n^{-}}(4 a d)^{|j-i|_{1}} a^{|i-n|_{1}}
$$

$$
\begin{aligned}
\sum_{j=0}^{n^{-}}\left|c_{j}^{n}\right| & \leqq \sum_{j}(4 a d)^{|j|_{1}} \sum_{i \neq 0} a^{|i|_{1}} \\
& \leqq \text { const } a
\end{aligned}
$$

where the constant is independent of $n$. This proves Part (a) of Lemma 3 for $a$ sufficiently small. Similarly from (3.12)

$$
\sum_{n>j}\left|c_{j}^{n}\right| \leqq \sum_{i, n}(4 a d)^{|i|_{1}} a^{|n|_{1}}=D<\infty
$$

where $D$ is independent of $j$. This proves part (b) of Lemma 3.

\section{References}

1. Abramowitz, N., Stegun,I.: Handbook of mathematical functions, pp. 375, 378, Washington: U.S. Govt. Printing Office 1964

2. Cannon, J.: Continuous sample paths in quantum field theory, to appear in Commun. math. Phys.

3. Colella, P., Lanford, O.: Sample field behavior for the free Markov random field. In: Velo, G., Wightman,A. (Ed.): Constructive quantum field theory, Springer Lecture Notes in Physics 25. Berlin-Heidelberg-New York: Springer 1973

4. Dimock, J., Glimm, J.: Measures on the Schwartz distribution space and applications to quantum field theory, to appear

5. Gelfand, I., Shilov, G.: Generalized functions I, p. 288, New York: Academic Press 1964

6. Gelfand, I., Vilenkin, N.: Generalized functions IV, p. 329, New York: Academic Press 1964

7. Guerra,F., Rosen,L., Simon,B.: The $P(\phi)_{2}$ Euclidean quantum field theory as classical statistical mechanics, Ann. Math., to appear

8. Hida, T.: Stationary stochastic processes, p. 70, Princeton: Princeton University Press 1970

9. Loève, M.: Probability theory, New York: Van Nostrand 1960

10. Nelson,E.: Quantum fields and Markoff fields. In: Proceedings of the Summer Institute of Partial Differential Equations, Berkeley, 1971, A.M.S., Providence 1973

11. Nelson, E.: J. Funct. Anal. 12, $97-112$ (1973)

12. Nelson, E.: J. Funct. Anal. 12, $211-227$ (1973)

Communicated by A. S. Wightman

M. Reed

Mathematics Department

Princeton University

Princeton, N. J. 08540, USA

L. Rosen

Mathematics Department

University of Toronto

Toronto, Canada M5S 1A1 